\title{
MICRO ELECTRET POWER GENERATOR
}

\author{
Justin Boland*, Yuan-Heng Chao*, Yuji Suzuki**, Y.C. Tai* \\ *Department of Electrical Engineering, California Institute of Technology \\ 1200 E. California Blvd., Pasadena, CA 91125 \\ **Department of Mechanical Engineering, The University of Tokyo \\ Hongo 7-3-1, Bunkyo-ku, Tokyo 113-8656, JAPAN
}

\begin{abstract}
We present the first micromachined rotational electret power generator, linearized theoretical model of electret power generation, and novel method to produce uniformly charged electret. We also improved our previously developed $[1,2]$ thin film Teflon AF 1601-S electret technology with respect to dielectric thickness, charge uniformity, and processability. In demonstration, our prototype power generator successfully generated $>25 \mu \mathrm{W}$ with electret thickness of $9 \mu \mathrm{m}$, effective charge density of $-2.8 \times 10^{-4} \mathrm{C} / \mathrm{m}^{2}$, and rotational speed of 4170 RPM.
\end{abstract}

\section{Introduction}

Electret generators differ from electromagnetic generators in that the electromotive force is purely electric. Electret generator theory and experiment was first reported by Jefimenko [3], and later refined by Tada [4]. An electret generator with a radius of $45 \mathrm{~mm}$ was studied by Tada [5]. Maximum reported power output from an electret generator was $1.02 \mathrm{~mW}$. The keys to miniaturizing this technology are micromachining and a compatible electret technology. By optimizing the micromachined power generator, maximum power generated can be greater than $1 \mathrm{~mW}$.

As an electret, Teflon can contain charge densities of $-5 \times 10^{-4} \mathrm{C} / \mathrm{m}^{2}$ with theoretical lifetimes of hundreds of years [6]. Previous work on micro electret microphones in this lab used Teflon AF 1601-S because it is a spin-on dielectric compatible with MEMS process. We extended our processing capabilities to allow for multiple spins of this material and also patterning using photoresist. Electrons can then be quickly implanted utilizing a back lighted thyratron (BLT) [7], also called a psuedospark device in literature [8].

\section{Design and Fabrication}

Rotors were made with a radius of $4 \mathrm{~mm}$ and stators with a radius of $5 \mathrm{~mm}$. Design size was chosen to maximize available area on a $1 \mathrm{~cm}^{2}$ chip to avoid having to stitch the exposures on the stepper. The rotor is only $4 \mathrm{~mm}$ in radius so that surface contact to the ground layer of the stator is possible with silver paste. Since only regions where the rotor and stator overlap result in the production of electricity, for all practical purposes, $r_{\text {eff }}=4 \mathrm{~mm}$ is used.

The number of poles in our experiments, $n=4$, were chosen to compare with results found in literature. In Tada's work, the number of poles remains low due to the method of making them, namely cutting by hand. MEMS lithography is capable of producing lines smaller than $10 \mu \mathrm{m}$, which far exceeds the assumptions that fringing fields can be neglected.

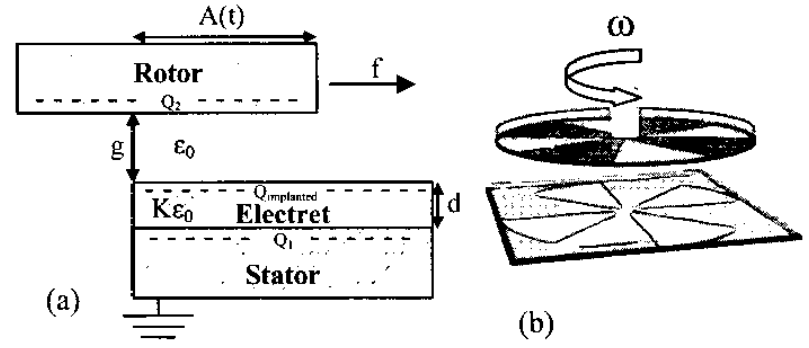

Figure 1. Schematics of electret generator (a) cross-section view (b) 3-D view showing a 4-pole rotor and stator

Teflon thickness for the generator was $9 \mu \mathrm{m}$, and, in contrast to Tada's setup, was on the stator. This configuration was chosen for the ability to try many different thicknesses without having to remount the rotor. The rotor must be mounted with plane normal aligned to the long axis of the axle or else the planes of the rotor and stator cannot be parallel during rotation. The dimensions can be easily seen in Figure 1.

Figure 2 shows the process flow of a rotor and stator with dielectric. Rotors and stators for electret generators should have a matching number of poles. For the rotor, $2000 \AA$ aluminum was evaporated onto a quartz wafer and then patterned. The wafer was then diced, and one die was diced into an octagonal shape to closer approximate a circular rotor. Stators are produced by first evaporating $2000 \AA$ aluminum onto a quartz wafer. The aluminum layer is patterned and then a thick layer of Teflon AF 1601-S is spun on.



In previous processing, it was determined that a $1.2 \mu \mathrm{m}$ Teflon layer can be spun-on if the Teflon solution is $6 \%$ solids and $94 \%$ Fluorinert FC-75, as supplied by Dupont. This thin 
film initially has a rough surface and after a long prebake at $330^{\circ} \mathrm{C}$ for 15 minutes to allow the surface to reflow. Baking at this temperature also has the added effect of removing all solvent, which is a necessary step when spinning multiple layers of Teflon.

Dupont also supplies an $18 \%$ solids version of the Teflon $\mathrm{AF}$, but this solution is too viscous for conventional spinning. We made a $7.4 \%$ solids mixture by mixing the $18 \%$ solids version of Teflon with Fluorinert FC-40. This solution produces spun-on films $9 \mu \mathrm{m}$ thick at 500RPM. Fluorinert FC40 has similar electrical characteristics to Fluorinert FC-75, but $\mathrm{FC}-40$ has a kinematic viscosity 2.75 times higher than FC-75. Furthermore, the $1.2 \mu \mathrm{m}$ film had height fluctuations greater than $25 \%$ while the $9 \mu \mathrm{m}$ film had variations less than $1 \%$. The main disadvantage of FC-40 is its higher boiling point, which means higher temperatures and longer bake times are required to drive off all solvent from the thicker film Teflon film.

Applying HMDS vapor for 3 minutes to the fully baked, spun-on Teflon modified its naturally hydrophobic nature enough for photoresist to be spun on top of the Teflon. Further trials also proved that spinning Teflon on fully baked Teflon is also possible with use of HMDS. The adhesion between Teflon layers appears to be very good, and often was better than adhesion between thermally evaporated aluminum and the substrate. In the case of a floating metal layer, adhesion between the aluminum that was evaporated on top of Teflon is sufficient unless the any part of the Teflon-aluminum interface is exposed to solvents. Thus, floating metal layers must be sealed before wet dicing or other wet etch steps occur.

\section{Floating Layer Electret}

Electron beam implantation is a well-studied method for implanting electrons within dielectrics. Beam writing can be performed by raster scanning over a dielectric; it takes considerable of time to implant a sufficient number of electrons while occupying an expensive machine for a menial task using this method. In contrast, a BLT provides a pulsed electron source with very large electron doses within $\sim 100 \mathrm{~ns}$. Implantation with the BLT produces a Gaussian distribution over the surface of the electret, as in Figure 3 (a), which is not desirable for providing a uniform electret. To alleviate this problem, a metal layer is deposited on top of a thick dielectric layer, pattemed to be electrically floating [9], and then sealed with a thin dielectric layer. The floating metal layer provides a reference voltage and therefore an electric field nonuniformity of less than $1 \%$ of the surface as seen in Figure 3 (b). At the time of submission of this paper, power generation experiments had not been performed using the floating layer electret because of difficulty with over-charging the floating metal and subsequent breakdown.
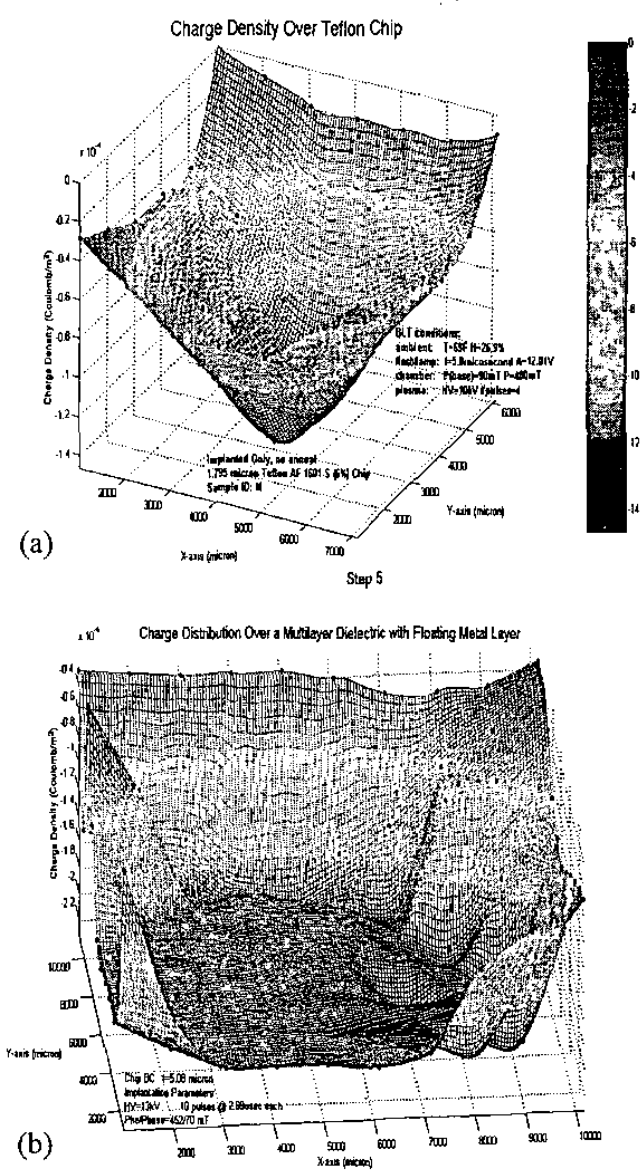

Figure 3. (a)Charge density of implanted Teflon using the back lighted thyratron (b) charge implanted in a chip with floating metal layer patterned into a circle, charge outside the metal circle is approximately equal to the Gaussian case

\section{Experimental Results}

We measure charge densities with a Monroe Electronics isoprobe Model 244 with a high resolution 1024AEH probe. We mounted the probe on an $x-y-z$ stage to allow precise measurements of the effective surface charge. Minimum observed resolution in $\mathrm{x}$ and in $\mathrm{y}$ was $244 \mu \mathrm{m}$, although the resolution of the stage was $25.4 \mu \mathrm{m}$ in $\mathrm{x}$-axis and $10 \mu \mathrm{m}$ in the $y$-axis. The electret generator relies on an electric field that is fixed in $z$ but variable in $x-y$, and therefore effective surface charge densities in $x-y$ defined by only the dielectric thickness and the voltage of the surface measured with the isoprobe is sufficient for quantifying the charge implanted.

After fabrication of the rotor and stator it is necessary to mount them to an apparatus that can supply rotation. We built a testbed for this purpose (Figure 4) with an angular misalignment of $0.46^{\circ}$ for the rotor, which was measured by shining a laser pointer at the spinning rotor and measuring the radius of the reflected circle and the baseline distance. A 5- 
axis micropositioner is used for aligning the stator to the rotor. In trying to minimize the gap spacing, the stator is lightly crashed into the rotor at one point, but because of angular misalignment the far end of the rotor is at least $80 \mu \mathrm{m}$ away from the stator.

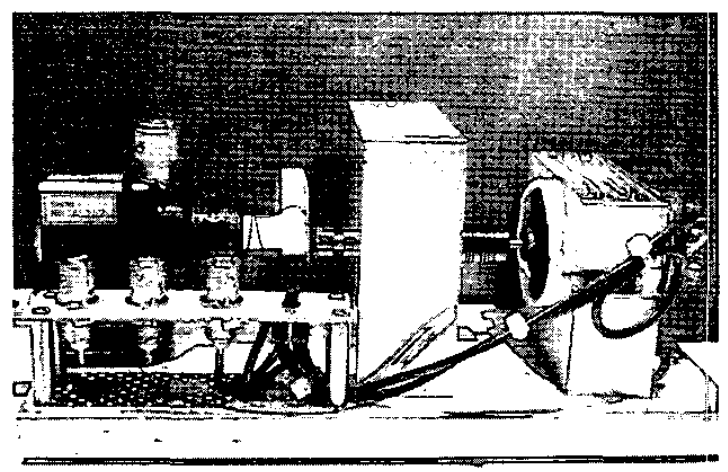

Figure 4. Testbed with rotor and stator mounted.

Power generation experiments using the testbed involves setting the gap distance, driving the motor at different speeds, and simultaneous measurement of speed and power output. The ground lead of the generator is the ground of the stator and the power lead is the chassis of the testbed which is electrically connected to the rotor through a bearing. The power lead is connected to a simple op-amp, National Semiconductor LF356, in a voltage follower configuration with $10^{12} \mathrm{Ohm}$ input impedance. This high impedance allows load matching by placing different load resistors across the power and ground. Power output is measured by two different means: (a) voltage output from the amplifier is fed to an HP $54503 \mathrm{~A} 500 \mathrm{MHz}$ Digitizing Oscilloscope to observe the waveform or (b) voltage output from the amplifier is measured in $V_{\text {RMS }}$ with a Fluke 87III True RMS handheld multimeter. Power from the generator is simply $\mathrm{V}_{\mathrm{RMS}}{ }^{2} / \mathrm{R}_{\mathrm{L}}$.

Several methods of measuring the speed were employed to check for accuracy. A stroboscopic tachometer showed some drift from other measurement techniques, so the output waveform from the 4-pole generator was used directly by measuring $n=4$ periods of the output signal. The motor is a 6-pole motor, and confirmation of speed measurements was made by connecting a secondary channel of the oscilloscope across the terminals of the motor and verifying that 6 periods of back-emf of the motor corresponded to 4 periods of the generator. Additionally, the Fluke handheld multimeter has an option to measure the frequency of an ac signal, which, as expected, was exactly 4 times larger than the frequency acquired from the other methods. The oscilloscope was the primary source of speed measurements. Pulse width modulation was not a viable option to control speed since the motor used draws a current up to $30 \mathrm{~A}$.

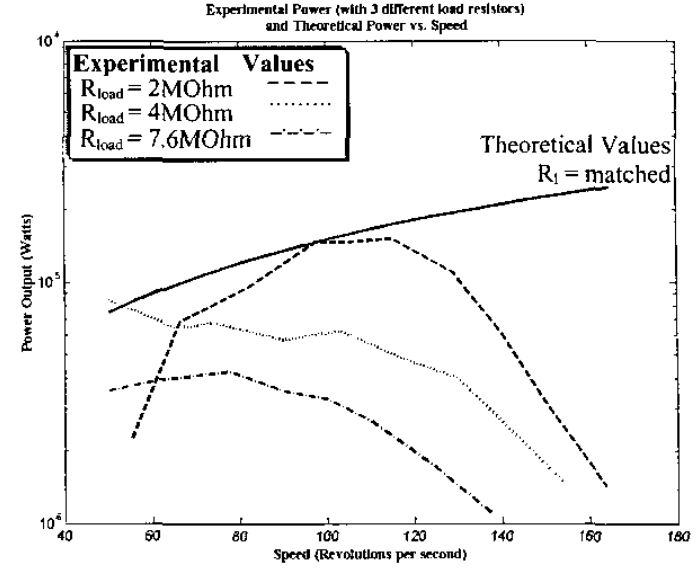

Figure 5. Theoretical values of a continuously load matched system and power output from 3 experimental trials using different load resistances

\section{Theory}

Assuming the width of the electrodes is large compared to the distance between them, a linearized theory is derived by assuming that an electret generator acts as a fixed-charge, variable capacitance device. Figure 1 explains the geometry used in the derivation.

Conservation of charge implies

$$
\mathrm{Q}_{\text {implanted }}=\mathrm{Q}_{1}(\mathrm{t})+\mathrm{Q}_{2}(\mathrm{t})
$$

Charge on capacitor is related to the area of the overlapping capacitors.

$$
\begin{aligned}
& \mathrm{C}_{1}(\mathrm{t})=\frac{\mathrm{K}_{\text {teflon }} \varepsilon_{0}}{\mathrm{~d}} \mathrm{~A}(\mathrm{t}) \\
& \mathrm{C}_{2}(\mathrm{t})=\frac{\varepsilon_{0}}{\mathrm{~g}} \mathrm{~A}(\mathrm{t})
\end{aligned}
$$

The equation describing the equivalent circuit is

$$
\begin{aligned}
& V(t)=\frac{-Q_{1}(t)}{C_{1}(t)}+\frac{Q_{2}(t)}{C_{2}(t)} \\
& =\left(\frac{-d}{K_{\text {teflon }} \varepsilon_{0}}\right) \frac{Q}{A}+\left(\frac{d}{K_{\text {teflon }} \varepsilon_{0}}+\frac{g}{\varepsilon_{0}}\right) \frac{Q_{2}(t)}{A(t)}
\end{aligned}
$$

Where $\mathrm{K}_{\text {tefion }}$ is the dielectric constant of Teflon AF 1601 listed as 1.93. Since

$$
\begin{aligned}
& \mathrm{V}(\mathrm{t})=\mathrm{IR}=-\frac{\partial \mathrm{Q}_{2}(\mathrm{t})}{\partial \mathrm{t}} \mathrm{R} \\
& \mathrm{I}(\mathrm{t})=\frac{\sigma \mathrm{d}}{\mathrm{K}_{\text {teflon }} \varepsilon_{0} \mathrm{R}}-\left(\frac{\mathrm{d}}{\mathrm{K}_{\text {teflon }} \varepsilon_{0}}+\frac{\mathrm{g}}{\varepsilon_{0}}\right) \frac{\mathrm{Q}_{2}(\mathrm{t})}{\mathrm{A}(\mathrm{t}) \mathrm{R}}
\end{aligned}
$$


$A(t)=\left\{\begin{array}{l}\frac{n \pi r^{2} f}{2} t \text { for } t: 0<t<\frac{1}{2 n f} \\ -\frac{n \pi r^{2} f}{2} t \text { for } t: \frac{1}{2 n f}<t<\frac{1}{n f}\end{array}\right.$

Let $\alpha=\left(\frac{\mathrm{d}}{\mathrm{K}_{\text {teflon }} \varepsilon_{0}}+\frac{\mathrm{g}}{\varepsilon_{0}}\right) \frac{1}{\mathrm{n} \pi \mathrm{r}^{2} \mathrm{fR}}$ and

$\beta=\frac{\sigma d}{\mathrm{~K}_{\text {teflon }} \varepsilon_{0} \mathrm{R}}$

Then $\mathrm{Q}_{2}(\mathrm{t})=\frac{\beta \mathrm{t}}{1+\alpha}-\mathrm{Ct}^{-\alpha}$

With capacitor plates completely out of phase at $t=0, Q_{2}(0)=0$

$$
\mathrm{I}(\mathrm{t})=\frac{-\sigma \frac{\mathrm{d}}{\varepsilon_{0}}}{\mathrm{R}+\frac{\mathrm{l}}{\mathrm{n} \pi \mathrm{r}^{2} \mathrm{f}}\left(\frac{\mathrm{d}}{\mathrm{K}_{\text {teflon }} \varepsilon_{0}}+\frac{\mathrm{g}}{\varepsilon_{0}}\right)}
$$

Maximum power is achieved when

$$
\mathrm{R}_{\text {optimal }}=\frac{\mathrm{l}}{\mathrm{n} \pi \mathrm{r}^{2} \mathrm{f}}\left(\frac{\mathrm{d}}{\mathrm{K}_{\text {teflon }} \varepsilon_{0}}+\frac{\mathrm{g}}{\varepsilon_{0}}\right)
$$

This gives a load-matched power equation

$$
\mathrm{P}_{\text {optimal }}=\frac{\sigma^{2} \mathrm{n} \pi \mathrm{r}^{2} \mathrm{f}}{\frac{4 \mathrm{~K}_{\text {teflon }} \varepsilon_{0}}{\mathrm{~d}}\left(1+\frac{\mathrm{K}_{\text {teflon }} \mathrm{g}}{\mathrm{d}}\right)}
$$

Charge density is limited by the dielectric strength of the material. In the case of Teflon AF $1601-S$, this value is $20 \mathrm{~V} / \mu \mathrm{m}$. Power output increases with decreasing dielectric constant, which is why Teflon AF with dielectric constant of 1.93 is chosen

Gap spacing (g) should be minimized but spacing smaller than $1 / 4$ of the dielectric thickness is sufficiently small. Therefore, gap spacing is directly related to the thickness of the electret. The thickness of the electret is limited by processing issues for Teflon AF, but if this were not the case then the limiting thickness is related to the breakdown voltage in air.

\section{Discussion}

Power generation experiments were performed and the results are shown in Figure 5. The experimental curve shown is a load matched curve (Equation 9) and uses a gap spacing of $60 \mu \mathrm{m}$. This is very reasonable considering that the minimum spacing is zero at the crashed edge and $80 \mu \mathrm{m}$ at the far edge. The other parameters used in the theoretical values match the measured values of the generator, which are $n=4, r=4 \mathrm{~mm}$, $\mathrm{s}=-2.8 \times 10^{-4} \mathrm{Coulomb} / \mathrm{m}^{2}, \mathrm{~K}_{\text {Teflon }}=1.93, \mathrm{~d}=9 \mu \mathrm{m}$. The noise in the experimental graphs are exactly because the stator was crashed into the rotor. This was necessary to know the gap spacing exactly. The generator continues to perform well under this condition, despite some wear to the surfaces.

To verify that neglecting the fringing field is a valid assumption, we say that the smallest dimension within $90 \%$ of the active generator area must be ten times larger than the gap distance. Since $90 \%$ of the effective area of an $r=5 \mathrm{~mm}$ generator is outside $r=1.58 \mathrm{~mm}$, the shortest dimension $w$ (see Figure 6 ) is found to be $1.2 \mathrm{~mm}$ by using the number of poles and the law of cosines. Assuming $w$ must be ten times larger than $\mathrm{g}$ and we previously stated that a decent $\mathrm{g}$ is preferably $1 / 4 \mathrm{~d}$, we get that $\mathrm{w}$ need only be $22.5 \mu \mathrm{m}$ for a $9 \mu \mathrm{m}$ dielectric thickness. The condition is more than met in our experiments, and by using this argument we expect to see good performance in generators with a few hundred poles.



Figure 6. Used to find the critical width $w$ from gap distance

\section{Conclusions}

Uniform charge density, gap control, and dielectric thickness are the primary challenges of designing and producing an electret generator. We engineered solutions to provide uniform charge density on thick, micromachinecompatible dielectric. We derived a linearized theory that adequately models experimental power measurements. Future work will focus on gap spacing, increasing the number of poles, elimination of rotor tilt, and verifying the charge distribution in the $z$-axis on charge implanted into a floating metal electret. We have already begun work on a testbed-less electret generator that overcomes the aforementioned difficulties by relying more heavily on the advantages of micromachining.

\section{References}

[1] T.Y. Hsu, W.H. Hsieh, K.Funutani and Y.C. Tai, Hilton Head 1996, pp. 235-238

[2] W.H. Hsieh, T.J. Yao and Y.C. Tai, MEMS 1999, pp. 1064-1067

[3] O.D. Jefimenko, IEEE Trans. Ind. Appl., Vol. IA-14, pp. 537-540, 1978

[4] Y. Tada, IEEE trans. Elect. Insul. EI-21, 1986, pp. 457-464

[5] Y. Tada, Jpn. J. Appl. Phys., Vol 31, Part 1, No. 3, 1992, pp. 846-851

[6] J.A. Malecki, Phys. Rev. B.. Vol. 59, no. 15, 1999, pp. 9954-9960

[7] T.Y. Hsu, "A Novel Electron Beam Source Based on the Back-Lighted Thyratron", Ph.D. dissertation, Univ. Southern California, 1992

[8] K. Frank, E. Dewald, C. Bickes, U. Emst, M. Iberler, J. Meier, U. Prucker, A. Rainer, M. Schlaug, J. Schwab, J. Urban, W. Weisser, and D. H. H. Hoffmann, IEEE trans. on Plasma Science, Vol. 27, No. 4, 1999, pp. 1008 1020

[9] Patent pending 